Research Article

\title{
Dendrobine Suppresses Lipopolysaccharide-induced Gut Inflammation in a Co-culture of Intestinal Epithelial Caco-2 Cells and RAW264.7 Macrophages
}

\author{
Huifan Liu, Jiaxi Liang, Gengsheng Xiao, Lukai Ma*, Qin Wang* \\ College of Light Industry and Food, Zhongkai University of Agriculture and Engineering, Guangzhou, Guangdong 510225, China
}

\section{ARTICLE INFO}

\section{Article History}

Received 12 January 2021

Accepted 09 April 2021

\section{Keywords}

Dendrobine

inflammatory bowel diseases

tight junctions

NF-kB pathway

\section{ABSTRACT}

We evaluated dendrobine for its potential effect on Inflammatory Bowel Disease (IBD) progression using the intestinal epithelial Caco-2/RAW264.7 macrophage co-culture cell model. The results showed that dendrobine maintained the tight junction (TJ) proteins in co-cultured Caco- 2 cells, suggesting its protective effect on intestinal integrity. Moreover, the findings indicated that the dendrobine group downregulated levels of tumor necrosis factor- $\alpha$ (TNF- $\alpha$ ), proinflammatory Interleukin (IL)- $1 \beta$ and IL-6, and inflammatory markers such as cyclooxygenase-2 (COX-2) and inducible nitric oxide synthase (iNOS) in co-cultured RAW264.7 cells. Additionally, dendrobine-mediated anti-inflammatory effects were observed based on the inhibition of LPSinduced proinflammatory mediators of nuclear factor kappa B (NF- $\kappa$ B)-related signaling. These results demonstrated the protective effect of dendrobine at effectively ameliorating intestinal barrier disruption and inhibiting activation of the NF- $\mathrm{B}$ pathway in macrophages through the intestinal barrier.

\section{GRAPHICAL ABSTRACT}

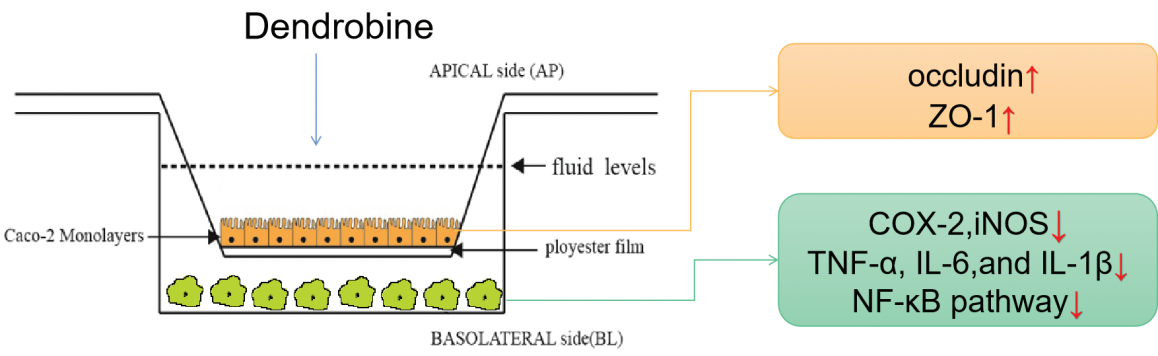

(C) 2021 The Authors. Publishing services by Atlantis Press International B.V. This is an open access article distributed under the CC BY-NC 4.0 license (http://creativecommons.org/licenses/by-nc/4.0/).

\section{INTRODUCTION}

Inflammatory Bowel Disease (IBD) is a type of chronic inflammation marked by the abnormal activation of macrophages in the intestinal lamina propria [1]. Intestinal Epithelial Cells (IECs) are part of this barrier and prevent the diffusion of pathogens into the circulatory system [2]. If the intestinal barrier is disrupted, intestinal permeability increases and immune homeostasis is disturbed, leading to intestinal diseases [3]. The Tight Junction (TJ) proteins occludin and Zonula Occludens Protein 1 (ZO-1) participate in maintaining intestinal structural integrity [4]. The mechanism underlying IBD causes increased intestinal inflammation and TJ permeability, which is associated with increased levels of antiinflammatory cytokines in the intestine and blood [5].

"Corresponding authors. Email: m1991lk@163.com; zhongkai_wq@126.com Peer review under responsibility of the International Association of Dietetic Nutrition and Safety
In vitro study of the intestinal epithelial barrier function requires the establishment and characterization of models reflecting the physiological relationships of cells. Many scholars have attempted to use co-culture models of IECs and immune cells to replicate their interactions in vitro [6]. The intestinal epithelial Caco-2 cell line has been widely used to simulate the intestinal monolayer due to their similarities in cell structure. Therefore, the use of Caco-2 cells in a co-culture with RAW264.7 macrophages represents a promising alternative to animal models for predicting the bioactive compounds associated with intestinal IBDs $[7,8]$.

Dietary ingredients, including flavonoids and phenolic chemicals, prevent TJ damage [2,9]. It has been implied that treatment with dietary bioactive compounds has the potential to prevent IBD [10]. Therefore, natural anti-inflammatory compounds, compared to conventional nonsteroidal anti-inflammatory drugs, are considered valuable based on their effective suppression of inflammation without causing side effects or inducing gastrointestinal toxicity [11]. 
Natural products, including those derived from Dendrobium, are recognized as sources of plant-based alkaloids and are in increasingly high demand by consumers and the food industry [12]. Dendrobium is a genus in the Orchidaceae family and is currently widely cultivated in Asia as a functional food source [13]. Dendrobine is an active alkaloid compound that has been found in Dendrobium [14]. Previous studies have shown that dendrobine can regulate biological functions due to its antitumor, antioxidant, and anti-inflammatory activities [15]. However, there are few reports of the effects of dendrobine on intestinal health, and thus an explanation of how dendrobine exerts its anti-inflammatory effect in this context is lacking. Therefore, we focused on the use of alkaloids to distinguish prevention from inflammation.

Here, we established a co-culture model, as follows. RAW264.7 macrophages were stimulated with Lipopolysaccharides (LPS) to induce inflammation, and Caco-2 cells were exposed to dendrobine. To investigate the regulation of the epithelial barrier by dendrobine, we examined the ability of dendrobine to increase Transepithelial Electrical Resistance (TEER) in the apical Caco-2 cell monolayer and evaluated changes in TJ protein levels by western blot. Furthermore, levels of inflammatory markers and Nuclear Factor Kappa B (NF-kB) pathway activation, which are both related to IBD, were evaluated following macrophage activation to assess the anti-inflammatory effects of dendrobine.

\section{MATERIALS AND METHODS}

\subsection{Materials}

A dendrobine standard (purity, >98\%) was obtained from the Shanghai Yuanye Biology Technique Co. Ltd. (Shanghai, China), and analytical-grade solvents and LPS were obtained from SigmaAldrich (Oakville, ON, Canada). Dulbecco's Modified Eagle Medium (DMEM) was obtained from Sigma-Aldrich, and Fetal Bovine Serum (FBS) and gentamycin were obtained from Gibco (Grand Island, NE, USA). Enzyme-linked Immunosorbent Assay (ELISA) kits for Nitric Oxide (NO), Tumor Necrosis Factor (TNF)- $\alpha$, Interleukin (IL)- $1 \beta$, IL-6, and Prostaglandin E (PGE2) were purchased from the Nanjing Jiancheng Institute of Biotechnology (Nanjing, China). RNAiso Plus (TRIzol) was obtained from Takara (Cat. \#9109; Shiga, Japan). Western blot analysis was performed using the following antibodies: anti-phosphorylated (p)-p65 (Cat. \#3033), anti-p65 (Cat. \#8242), anti-p-IкB $\alpha$ (Cat. \#2859), and antiI $\kappa \mathrm{B} \alpha$ (Cat. \#4812) from Cell Signaling Technology (Danvers, MA, USA). Anti-occludin (Cat. \#13409-1-ap) and anti-ZO-1 (Cat. \#21773-1-ap) were obtained from Proteintech (Rosemont, IL, USA). A BCA kit (AR0146) was obtained from Boster Biological Technology (Pleasanton, CA, USA). Polyvinylidene Difluoride (PVDF) membranes were obtained from Millipore (Merck KGaA, Darmstadt, Germany).

\subsection{Cell Culture}

The experiment was conducted according to the methods of a previous report [10], with some modifications. Caco-2 and RAW264.7 cells were obtained from the Shanghai Cell Bank/Stem Cell Bank at the Chinese Academy of Sciences (Shanghai, China). The cell line culture condition had a 5\% $\mathrm{CO}_{2}$ atmosphere using DMEM, with 10\% (v/v) FBS (Gibco). The RAW264.7 macrophages were seeded at a density of $5 \times 10^{5}$ cells per $\mathrm{cm}^{2}$, while the Caco- 2 cells were plated at density of $5 \times 10^{5}$ cells per $\mathrm{cm}^{2}$ on polycarbonate membranes (MillicellTM Culture Plate Inserts, $12 \mathrm{~mm}$ diameter) with a pore size of $0.4 \mu \mathrm{m}$ (Millipore). All experiments were performed on differentiated Caco- 2 cell monolayers at 21 days and a $24 \mathrm{~h}$ culture of RAW264.7 macrophages post-seeding.

\subsection{Sodium Fluorescein Assay}

A sodium fluorescein assay was used to verify the tightness of the Caco-2 monolayers. It was performed according to the methods of a previous study [16].

\subsection{TEER Measurement}

Transepithelial electrical resistance was measured by using a Millipore ERS-2 resistor (Millipore). Only monolayers with TEER values $>800 \Omega \cdot \mathrm{cm}^{2}$ were used in the experiments [7]. TEER values were measured following different treatments according to a previously described method [13].

\subsection{Cell Viability Assay}

Cell viability was measured at $24 \mathrm{~h}$ using an MTT assay to evaluate dendrobine cytotoxicity to Caco-2 and RAW264.7 cells according to a previously described method [13].

\subsection{Intestinal Anti-inflammatory Experiments}

To determine the intestinal anti-inflammatory potential of dendrobine, the Caco- 2 cell monolayers were pre-seeded for 21 days on the apical side and the RAW264.7 macrophages were pre-seeded for $24 \mathrm{~h}$ on the basolateral side in transwell plates. The experiment proceeded with the addition of the dendrobine standard ( 1 and $10 \mu \mathrm{g} / \mathrm{mL}$ ) to the apical side of the co-culture system at $37^{\circ} \mathrm{C}$ for $2 \mathrm{~h}$. The RAW264.7 cells on the basolateral side were then stimulated by the addition of $1 \mu \mathrm{g} / \mathrm{mL}$ LPS for $3 \mathrm{~h}$, according to a previously described method [10]. At the end of the incubation period, the basolateral culture media were collected for subsequent measurement. Caco-2 and RAW264.7 cells were then used for subsequent analysis.

\subsection{Measurement of Cytokines, PGE2, and NO Secretion}

Levels of IL-6, IL- $1 \beta$, TNF- $\alpha$, and PGE2 in the basolateral culture media were quantified using the ELISA kits according to the manufacturer's instructions. The NO level of the basolateral culture media was also quantified using the Griess method. Quantification was performed after calibration to the standards. 


\subsection{Quantitative Real-Time Polymerase Chain Reaction}

Total RNA was isolated from cells using TRIzol reagent according to the manufacturer's instructions. Equal amounts of total RNA from each sample were reverse transcribed to cDNA. The sequences of the primers are provided in Table 1 . The reaction mixtures were incubated for an initial denaturation at $50^{\circ} \mathrm{C}$ for $3 \mathrm{~min}$, followed by $95^{\circ} \mathrm{C}$ for $10 \mathrm{~min}$ and 40 cycles of $10 \mathrm{~s}$ at $95^{\circ} \mathrm{C}$ and $30 \mathrm{~s}$ at $60^{\circ} \mathrm{C}$. The relative amount of each gene was calculated using the $2^{-\Delta \Delta \mathrm{CT}}$ method.

\subsection{Western Blot}

Total protein was extracted using radioimmunoprecipitation assay buffer, and the protein concentrations were determined using the BCA kit. Proteins $(20 \mu \mathrm{g})$ were separated and subsequently transferred to PVDF membranes. The membranes were blocked with $5 \%$ bovine serum albumin containing $0.05 \%$ Tween-20 in Trisbuffered saline for $4 \mathrm{~h}$ at room temperature, followed by incubation with primary antibodies overnight at $4^{\circ} \mathrm{C}$. The membranes were then washed twice and incubated with secondary antibodies for $1 \mathrm{~h}$ at room temperature. $\beta$-actin was used as the loading control. Protein bands were detected by enhanced chemiluminescence.

Table 1 Primer sequences

\begin{tabular}{lll}
\hline Primer & Primer sequences $\left(\mathbf{5}^{\prime} \rightarrow \mathbf{3}^{\prime}\right)$ & NCBI reference \\
\hline iNOS-mF & GTTCTCAGCCCAACAATACAAGA & NM 010927.4 \\
iNOS-mR & GTGGACGGGTCGATGTCAC & \\
COX2-mF & TTCCAATCCATGTCAAAACCGT & NM 011198.4 \\
COX2-mR & AGTCCGGGTACAGTCACACTT & \\
IL- $1 \beta-\mathrm{mF}$ & GAAATGCCACCTTTTGACAGTG & NM 008361.4 \\
IL- $1 \beta-\mathrm{mR}$ & TGGATGCTCTCATCAGGACAG & \\
IL-6-mF & TAGTCCTTCCTACCCCAATTCC & NM 031168.2 \\
IL-6-mR & TTGGTCCTTAGCCACTCCTTC & \\
TNF- $\alpha-\mathrm{mF}$ & CTGAACTTCGGGGTGATCGG & XM 021149735.1 \\
TNF- $\alpha-\mathrm{mR}$ & GGCTTGTCACTCGAATTTTGAGA & \\
GAPDH-mF & GGTGAAGGTCGGTGTGAACG & XM 017321385.2 \\
GAPDH-mR & CTCGCTCCTGGAAGATGGTG & \\
\hline
\end{tabular}

GAPDH, Glyceraldehyde-3-phosphate dehydrogenase.

\subsection{Statistical Analysis}

All values are expressed as the mean \pm Standard Deviation (SD). Statistical analyses were performed using GraphPad Prism (version 7.03; San Diego, CA, USA). The results were analyzed via one-way Analysis of Variance (ANOVA) multiple comparisons; ${ }^{*} p<0.05$, ${ }^{* *} p<0.01,{ }^{* * *} p<0.001,{ }^{* * *} p<0.0001$, and NS means non-significant as compared with the control.

\section{RESULTS}

\subsection{Establishment of Caco-2 Monolayers}

The intestinal-like model was first established and verified before conducting subsequent experiments. TEER values increased with time following incubation, with all values at $\sim 800 \Omega \cdot \mathrm{cm}^{2}$ after the 21-day incubation, which suggested the formation of a TJ structure (Supplementary Figure S1A). Verification of Caco-2 monolayer formation indicated a sodium fluorescein absorptivity within 0-5\% (Supplementary Figure S1B), confirming its formation according to international standard terminology [13]. Furthermore, we observed no dendrobine cytotoxicity at 1 or $10 \mu \mathrm{g} / \mathrm{mL}$ (Supplementary Figure S1C). Hence, according to the tests above, the Caco-2 monolayers were formed successfully, and were subsequently prepared for establishing the co-culture model.

\subsection{Dendrobine Supports Intestinal Integrity}

A previous study indicated that pathogenesis of IBD increased intestinal permeability [4]. In the co-culture system, the LPS treatment group showed a 0.81 -fold increase in TEER relative to untreated controls $(p<0.05)$. The result showed that the intestinal inflammatory model was formed, leading to high intestinal permeability. Additionally, dendrobine treatment protected the Caco- 2 cell monolayer, as indicated by the fact that TEER values in the dendrobine treatment group were similar to those in controls (Figure 1A). The TEER values reflected that intestinal permeability was low after exposure with dendrobine.
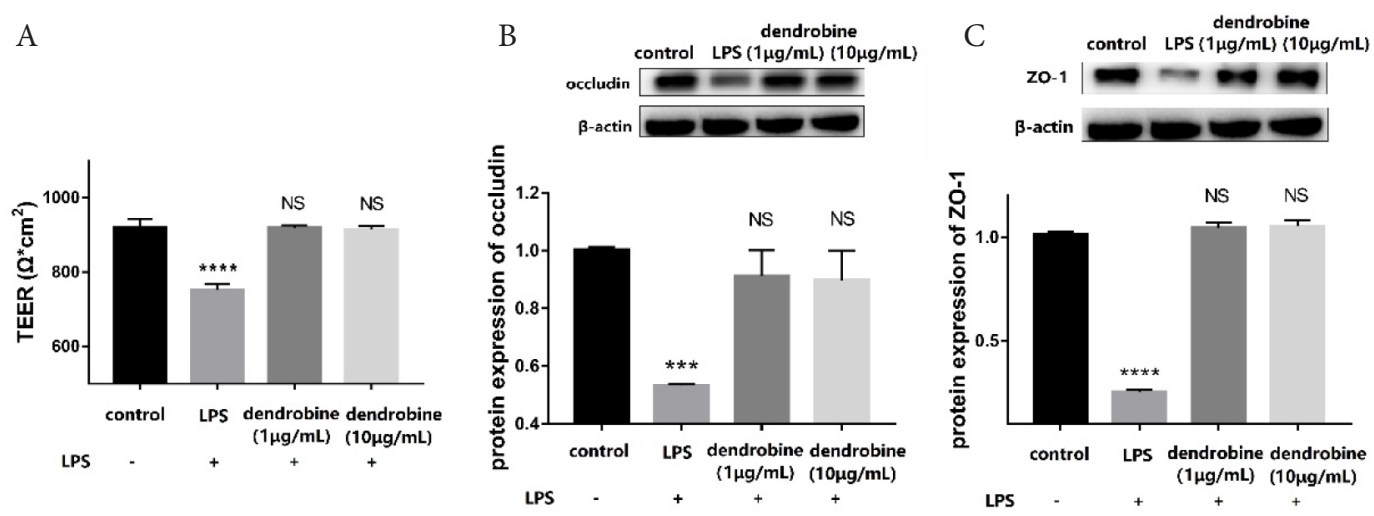

Figure 1 Dendrobine effects on TJs. (A) TEER values and (B and C) western blot results $(n=4)$. Levels of (B) occludin, (C) ZO-1 in Caco-2 cells (apical side) in the co-culture model. Each value represents the mean \pm SD. The results were analyzed via one-way ANOVA multiple comparisons; ${ }^{* * *} p<0.001,{ }^{* * * *} p<0.0001$, NS: non-significant was compared with control. 
The proteins forming the tight junctions between adjacent IECs comprise the basic unit of the intestinal epithelial barrier [2]. To determine whether dendrobine protects the in vitro Caco-2/ RAW264.7 model from LPS-induced disruption through regulation of TJs, we analyzed levels of ZO-1 and occludin in each experimental group. LPS treatment significantly downregulated occludin levels $(p<0.05)$, whereas dendrobine treatment inhibited this effect in Caco-2 cells (Figure 1B). Moreover, LPS treatment significantly decreased ZO-1 levels $(p<0.05)$ relative to the control, whereas dendrobine treatment significantly increased ZO-1 levels in Caco-2 cells $(p<0.05$; Figure $1 C)$. Dendrobine treatment at 1 and $10 \mu \mathrm{g} / \mathrm{mL}$ showed no significant difference with the control in ZO-1 and occludin expression $(p>0.05)$. These results are in agreement with those of previous reports that showed that bioactive compounds protect intestinal integrity by up-regulating the TJ proteins $[9,17]$. Therefore, a protective effect of dendrobine can be concluded, based on its regulation of TJ protein levels in Caco- 2 cells.

\subsection{Dendrobine Inhibits NO and PGE2 Production}

In inflammation, macrophages are activated, leading a series of mediator levels, such as NO and PEG2, to increase abnormally [18]. To investigate the potential anti-inflammatory effects of dendrobine, $\mathrm{NO}$ and PGE2 production and their relative protein expression

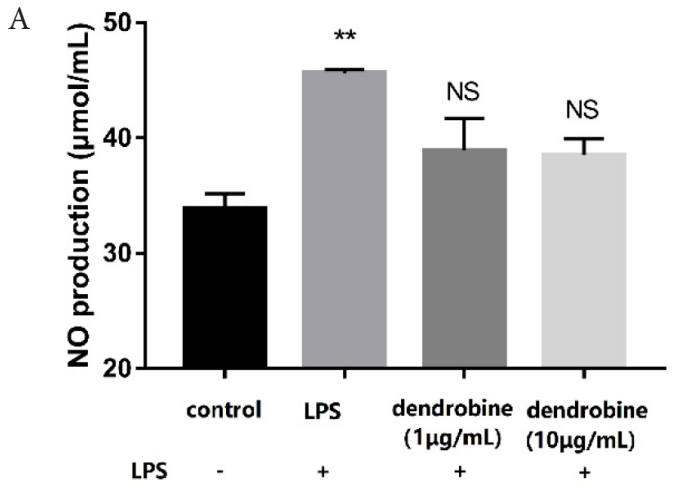

C

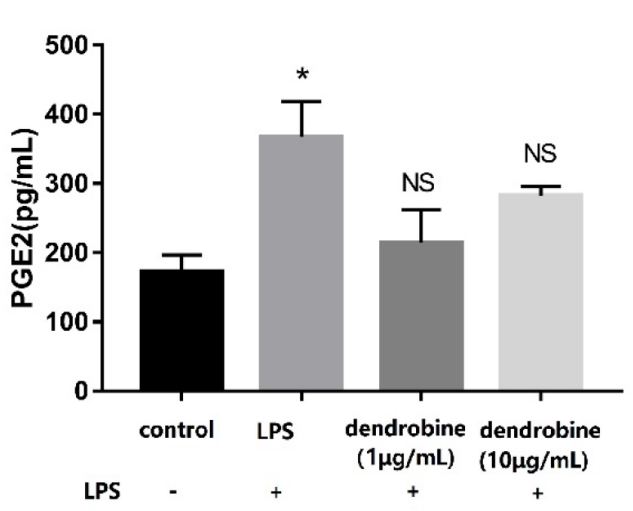

was determined. Inducible Nitric Oxide Synthase (iNOS) catalyzes NO production [19], which suggests that inhibition of excess NO production and expression of iNOS could be a therapy to prevent IBD. The results indicated that dendrobine $(10 \mu \mathrm{g} / \mathrm{mL})$ inhibited NO production (Figure 2A) based on a 0.84 -fold decrease in NO level relative to the LPS group. Additionally, when treating with dendrobine ( 1 and $10 \mu \mathrm{g} / \mathrm{mL}$ ) on the apical side, NO levels on the basolateral side were comparable with control levels (Figure 2A). As seen in Figure 2B, the levels of iNOS mRNA expression levels changed in a negative dose-dependent manner to the dendrobine concentration. Moreover, we found that dendrobine $(10 \mu \mathrm{g} / \mathrm{mL})$ resulted in a decrease in iNOS expression relative to that observed in LPS-treated cells ( $p<0.05$; Figure $2 \mathrm{~B}$ ). A likely explanation for the observed decrease in NO level could be concomitant downregulation of iNOS expression by LPS-activated macrophages.

Cyclooxygenase-2 (COX-2), induced by inflammatory stimulation, catalyzes arachidonic acid to produce prostaglandin, resulting in the secretion of large amounts of PGE2 and leading to intestinal inflammation [10]. On the basolateral side, the dendrobine group resulted in a decrease in PGE2 level relative to that observed in LPS-treated cells $(p<0.05$; Figure 2C). We found that dendrobine treatment exhibited anti-inflammatory activity according to attenuated COX-2 expression (Figure 2D). A previous study indicated that decreasing NO and PGE2 levels could be a way to alleviate inflammation [20]. The decrease in NO and PGE2 after treatment with dendrobine reflected an anti-inflammatory effect.
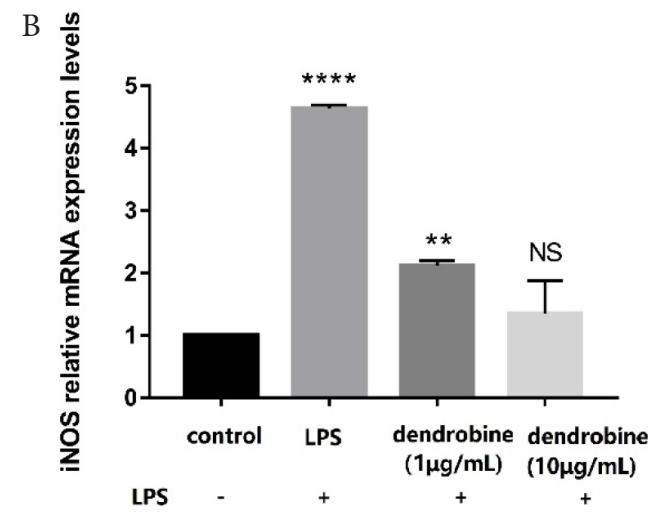

$\mathrm{D}$

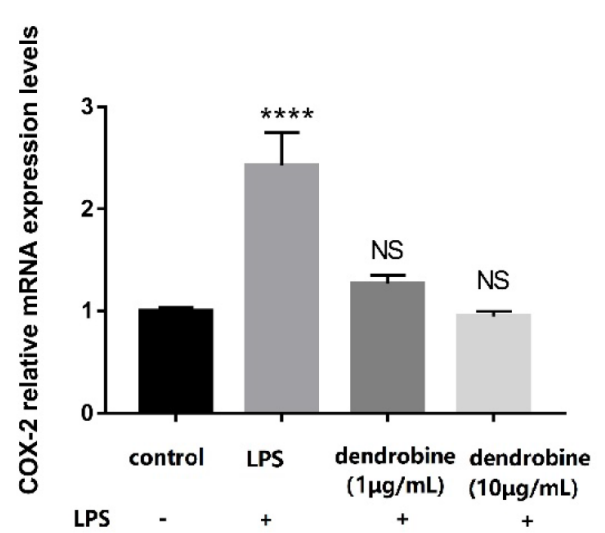

Figure 2 The effect of dendrobine on NO and PGE2 production and iNOS and COX-2 mRNA expression. (A) NO and (C) PGE2 production in co-cultured RAW264.7 macrophages (basolateral side) according to ELISA $(n=4)$. (B) iNOS and (D) COX-2 expression in co-cultured RAW264.7 macrophages (basolateral side) according to RT-PCR $(n=4)$. Each value represents the mean \pm SD. The results were analyzed via one-way ANOVA multiple comparisons; ${ }^{*} p<0.05,{ }^{* *} p<0.01,{ }^{* * *} p<0.0001$, NS: non-significant was compared with control. 


\subsection{Dendrobine Inhibits Cytokine Production}

Excess production of proinflammatory cytokines in macrophages may increase the permeability of the intestinal epithelium [1]. After LPS stimulation of RAW264.7 macrophages to mimic intestinal inflammation, we measured levels of proinflammatory cytokines (IL-1 $\beta$, IL- 6 , and TNF- $\alpha$ ) following dendrobine treatment (Figure 3). We found significantly decreased levels of TNF- $\alpha$ content, and the mRNA levels were lower in LPS-stimulated RAW264.7 cells treated with dendrobine $(p<0.05$; Figure $3 \mathrm{~A}$ and $3 \mathrm{C})$. The 0.52 -fold decrease in IL-1 $\beta$ mRNA expression in the dendrobine group $(10 \mu \mathrm{g} / \mathrm{mL})$ compared to the LPS group is shown in Figure 3F. Additionally, we observed that dendrobine $(10 \mu \mathrm{g} / \mathrm{mL})$ treatment dramatically decreased the IL-6 level compared to the LPS group in activated RAW264.7 cells (Figure 3B and 3E). There are some reports on the intestinal anti-inflammatory activity of alkaloids such as berberine [21] or oxymatrine [22] in decreasing the level of cytokines. Similarly, the results of the current study demonstrated that the addition of dendrobine in the apical side attenuated proinflammatory cytokine production in the basolateral side of the co-culture system.

\subsection{Dendrobine Inhibits the NF-KB Signaling Pathway in RAW264.7 Cells of the Co-culture Model}

Nuclear factor kappa B regulates proinflammatory mediators in stimulated macrophages causing subsequent upregulation of iNOS, COX-2, and proinflammatory cytokines such as TNF- $\alpha$. Additionally, activation of NF- $\mathrm{kB}$ is associated with the hyperphosphorylation and degradation of $\operatorname{I\kappa B} \alpha$ [20]. To elucidate the molecular mechanism of the dendrobine-mediated regulation of inflammation at the intestinal epithelial barrier, we evaluated its effect on the NF- $\mathrm{kB}$ pathway. We observed upregulated phosphorylation of the p65 subunit and IкB (according to increased p-p65/ p65 and p-IкB/IкB ratios) in LPS-stimulated RAW264.7 cells. However, treatment with dendrobine markedly $(p<0.05)$ decreased these ratios and downregulated the levels of both p-p65 and p-IкB (Figure 4). It is known that ameliorating the protein expression ratio of p-p65/p65 and p-IкB/IкB can suppress the NF- $\kappa B$ pathway [23]. Similarly, boldine is an alkaloid that suppresses IBD by targeting the NF- $\mathrm{kB}$ pathway [24]. The results of the current study indicate that dendrobine in the apical side alters the phosphorylation of proteins regulating NF- $\mathrm{KB}$ activity of macrophages in the basolateral side, thereby attenuating NF- $\mathrm{kB}$ signaling, which could possibly alleviate IBD symptoms.

\section{DISCUSSION}

As a traditional food and medicine, administration of Dendrobium allows dendrobine to exert its bio-functional effect in the body, especially exposure in gastrointestinal tract. The mechanism through which dendrobine exposure affects the inflamed intestine has hitherto been unclear. Therefore, we elucidated the underlying intestinal anti-inflammatory effect of dendrobine in this study.

Lipopolysaccharides-mediated intestinal inflammatory responses and the resulting damage to the intestinal TJ barrier from LPS are widely reported in studies of IBD. The disruption of TJ protein function induces IBD [17]. TJ proteins, acting as a natural intestinal barrier, prevent toxins and microbial antigens from damaging the lamina propria. A previous study showed that decreases in the levels of TJ proteins contributes to colitis [4]. To determine the TJ of IECs, the TEER was widely applied in the Caco-2 monolayer [25]. In the present study, this co-culture model showed that TEER decreased significantly following basolateral LPS treatment, but that dendrobine treatment upregulated levels of TJ proteins. These
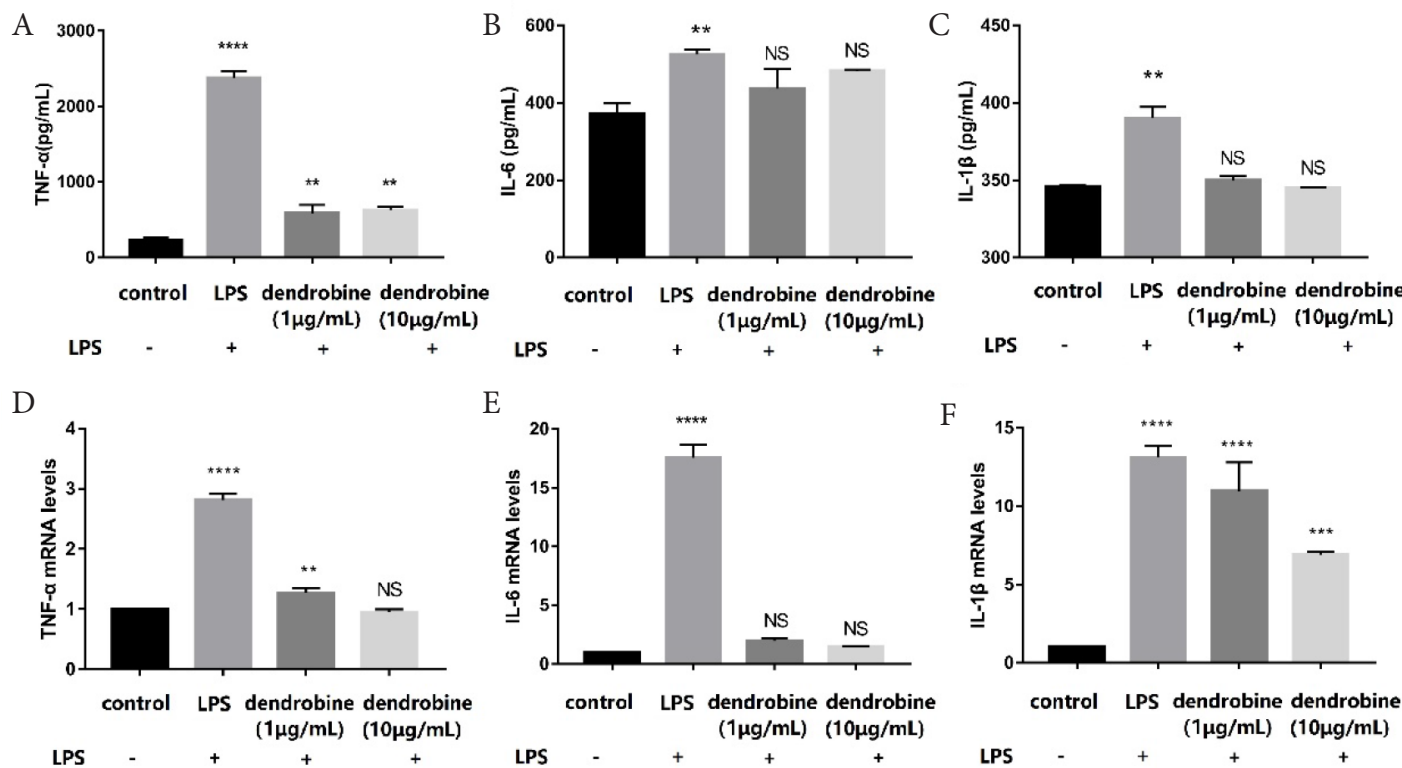

Figure 3 The effect of dendrobine on cytokine production (basolateral side). (A-C) Content and (D-F) mRNA levels of cytokines in RAW264.7 macrophages in the co-culture according to ELISA and qRT-PCR analyses, respectively $(n=4)$. Each value represents the mean \pm SD. The results were analyzed via one-way ANOVA multiple comparisons; ${ }^{* *} p<0.01,{ }^{* * *} p<0.001,{ }^{* * * *} p<0.0001$, NS: non-significant was compared with control. 
results suggest that dendrobine supports intestinal barrier integrity by regulating TJ protein production.

Previous studies have demonstrated that changes in intestinal epithelial monolayer permeability are directly related to the alteration of TJs, and demonstrating the importance of cytokines and TJ proteins in securing the intestinal barrier [2,9]. TNF- $\alpha$ is produced mainly by activated macrophages, monocytes, and $\mathrm{T}$ cells and is one of the major cytokines associated with IBD [26]. The imbalance of cytokines may affect inflammation, including IBD [27]. Inhibition of NF- $\kappa \mathrm{B}$ activation is an effective strategy to prevent the production of excess cytokines [28]. In this study, the levels of cytokines (IL-6, TNF- $\alpha$, and IL- $1 \beta$ ) and anti-inflammatory mediators (NO and PGE2) decreased in the dendrobine treatment group. Further, we found that LPS treatment increased the phosphorylation of regulators of NF- $\kappa \mathrm{B}$-mediated proinflammatory signaling, whereas dendrobine treatment decreased these levels and inhibited NF- $\kappa \mathrm{B}$ activation in RAW264.7 cells while also upregulating TJ protein levels in Caco-2 cells (Figure 5).

Similarly, it has been reported that other natural compounds, such as purple carrot anthocyanins, Sambucus nigra fruit extract, and

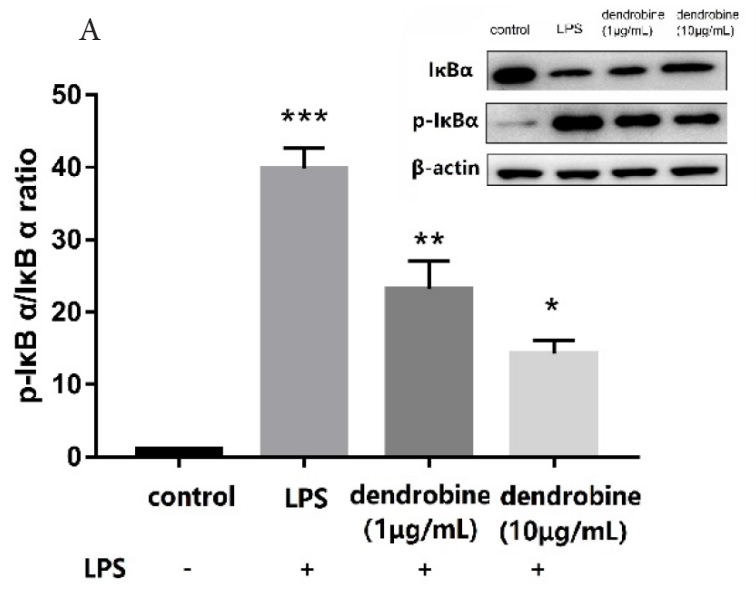

B

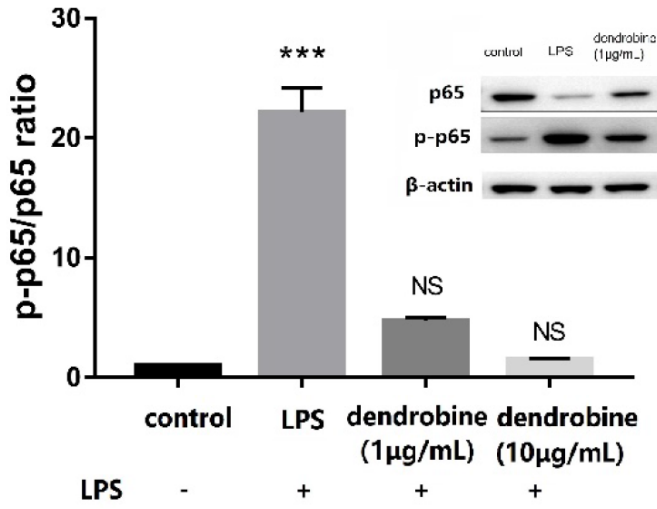

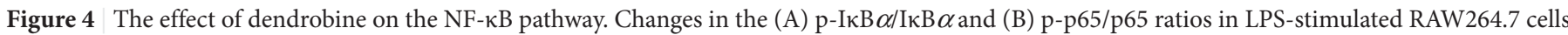
(basolateral side) after dendrobine treatment according to western blot $(n=4)$. Each value represents the mean \pm SD. The results were analyzed via oneway ANOVA multiple comparisons; ${ }^{*} p<0.05,{ }^{* *} p<0.01,{ }^{* * *} p<0.001$, NS: non-significant was compared with control.

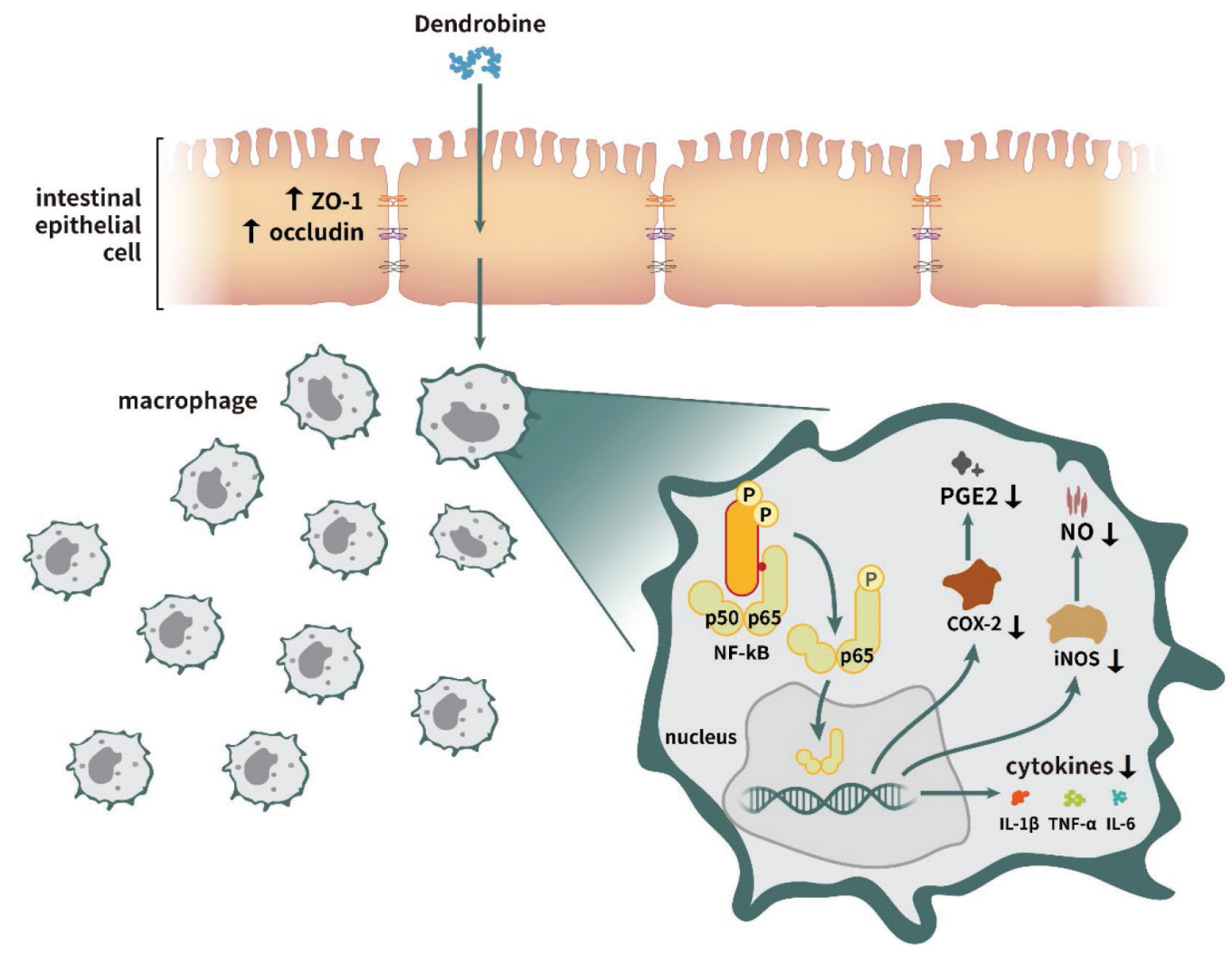

Figure 5 Proposed dendrobine-mediated intestinal anti-inflammatory mechanism. 
Kaempferol can directly or indirectly mitigate inflammation in RAW264.7 cells through the Caco-2 monolayer [2,7,10]. These examples support our results that dendrobine, as a natural compound, decreases the inflammatory level in the co-culture model. Therefore, dendrobine might directly or indirectly prevent the secretion of proinflammatory cytokines in RAW264.7 macrophages by the NF- $\kappa$ B pathway, and inhibiting protection of TJs between intestinal epithelial cells.

\section{CONCLUSION}

In summary, these findings indicate that dendrobine, as a natural bioactive compound, may be a potential supplement to prevent IBD-related diseases. Further study will investigate its bioavailability to determine the most effective concentration of dendrobine in vivo. These results will help support the common functional beverage industry and the development of foods containing these bioactive compounds.

\section{CONFLICTS OF INTEREST}

The authors declare they have no conflicts of interest.

\section{AUTHORS' CONTRIBUTION}

HL designed the study, analyzed and interpreted the data. JL prepared the paper. LM performed the experiments and interpreted the data. QW provided analysis for the study. GX interpreted the findings.

\section{FUNDING}

This study was funded by the Basic Research and Applied Basic Research Funding Project of Guangdong Province (Grant No. 2019A1515011283) and Guangzhou Science and Technology Commissioner Project (Project No. GZKTP201937).

\section{ABBREVIATIONS}

COX-2, cyclooxygenase-2; DMEM, Dulbecco's modified Eagle medium; ELISA, enzyme-linked immunosorbent assay; FBS, fetal bovine serum; GC, gas chromatography; IBD, inflammatory bowel disease; IEC, intestinal epithelial cell; IL, interleukin; iNOS, inducible nitric oxide synthase; LPS, lipopolysaccharide; MS, mass spectrometry; NF- $\kappa \mathrm{B}$, nuclear factor kappa B; NO, nitric oxide; PVDF, polyvinylidene difluoride; RT-PCR, polymerase chain reaction; TJ, tight junction; TNF, tumor necrosis factor; PGE, prostaglandin E; TEER, transepithelial electrical resistance; ZO-1, zonula occludens protein 1.

\section{SUPPLEMENTARY MATERIALS}

Supplementary data related to this article can be found at https:// doi.org/10.2991/efood.k.210409.001.

\section{REFERENCES}

[1] Tanoue T, Nishitani Y, Kanazawa K, Hashimoto T, Mizuno M. In vitro model to estimate gut inflammation using co-cultured Caco-2 and RAW264.7 cells. Biochem Biophys Res Commun 2008;374:565-9.

[2] Bian Y, Dong Y, Sun J, Sun M, Hou Q, Lai Y, et al. Protective effect of Kaempferol on LPS-induced inflammation and barrier dysfunction in a coculture model of intestinal epithelial cells and intestinal microvascular endothelial cells. J Agric Food Chem 2020;68:160-7.

[3] Kim MS, Kim JY. Intestinal anti-inflammatory effects of cinnamon extracts in a co-culture model of intestinal epithelial Caco-2 cells and RAW264.7 macrophages. Appl Biol Chem 2017;60:553-61.

[4] Zhou X, Qi W, Hong T, Xiong T, Gong D, Xie M, et al. Exopolysaccharides from Lactobacillus plantarum NCU116 regulate intestinal barrier function via STAT3 signaling pathway. J Agric Food Chem 2018;66:9719-27.

[5] Neurath MF. Cytokines in inflammatory bowel disease. Nat Rev Immunol 2014;14:329-42.

[6] Ding X, Yu Q, Hou K, Hu X, Wang Y, Chen Y, et al. Indirectly stimulation of DCs by Ganoderma atrum polysaccharide in intestinal-like Caco-2/DCs co-culture model based on RNA-seq. J Funct Foods 2020;67:103850.

[7] Olejnik A, Kowalska K, Olkowicz M, Rychlik J, Juzwa W, Myszka $\mathrm{K}$, et al. Anti-inflammatory effects of gastrointestinal digested Sambucus nigra L. fruit extract analysed in co-cultured intestinal epithelial cells and lipopolysaccharide-stimulated macrophages. J Funct Foods 2015;19:649-60.

[8] Hu X, Yu Q, Hou K, Ding X, Chen Y, Xie J, et al. Regulatory effects of Ganoderma atrum polysaccharides on LPS-induced inflammatory macrophages model and intestinal-like Caco-2/ macrophages co-culture inflammation model. Food Chem Toxicol 2020;140:111321.

[9] Liu P, Bian Y, Fan Y, Zhong J, Liu Z. Protective effect of naringin on in vitro gut-vascular barrier disruption of intestinal microvascular endothelial cells induced by TNF- $\alpha$. J Agric Food Chem 2020;68:168-75.

[10] Olejnik A, Kowalska K, Kidoń M, Czapski J, Rychlik J, Olkowicz $\mathrm{M}$, et al. Purple carrot anthocyanins suppress lipopolysaccharideinduced inflammation in the co-culture of intestinal Caco-2 and macrophage RAW264.7 cells. Food Funct 2016;7:557-64.

[11] McDonald DD. Predictors of gastrointestinal bleeding in older persons taking nonsteroidal anti-inflammatory drugs: results from the FDA adverse events reporting system. J Am Assoc Nurse Pract 2019;31:206-13.

[12] Liu H, Ma J, Gong F, Wei F, Zhang X, Wu H. Structural characterisation and immunomodulatory effects of polysaccharides isolated from Dendrobium aphyllum. Int J Food Sci Technol 2018;53:1185-94.

[13] Liu H, Ye H, Sun C, Xi H, Ma J, Lai F, et al. Antioxidant activity in HepG2 cells, immunomodulatory effects in RAW 264.7 cells and absorption characteristics in Caco-2 cells of the peptide fraction isolated from Dendrobium aphyllum. Int J Food Sci Technol 2018;53:2027-36.

[14] Wang S, Wu H, Geng P, Lin Y, Liu Z, Zhang L, et al. Pharmacokinetic study of dendrobine in rat plasma by ultraperformance liquid chromatography tandem mass spectrometry. Biomed Chromatogr 2016;30:1145-9. 
[15] Yang D, Cheng ZQ, Yang L, Hou B, Yang J, Li XN, et al. Secodendrobine-type alkaloids and bioactive phenolics from Dendrobium findlayanum. J Nat Prod 2018;81:227-35.

[16] Wu H, Xi H, Lai F, Ma J, Chen W, Liu H. Cellular antioxidant activity and Caco-2 cell uptake characteristics of flavone extracts from Labisia pumila. Int J Food Sci Technol 2019;54:536-49.

[17] Chen M, Liu Y, Xiong S, Wu M, Li B, Ruan Z, et al. Dietary L-tryptophan alleviated LPS-induced intestinal barrier injury by regulating tight junctions in a Caco-2 cell monolayer model. Food Funct 2019;10:2390-8.

[18] Hwang D, Kang MJ, Jo MJ, Seo YB, Park NG, Kim GD. Antiinflammatory activity of $\beta$-thymosin peptide derived from Pacific oyster (Crassostrea gigas) on $\mathrm{NO}$ and $\mathrm{PGE}_{2}$ production by down-regulating NF- $\kappa B$ in LPS-induced RAW264.7 macrophage cells. Mar Drugs 2019;17:129.

[19] Wang Q, Liang J, Brennan CS, Ma L, Li Y, Lin X, et al. Antiinflammatory effect of alkaloids extracted from Dendrobium aphyllum on macrophage RAW 264.7 cells through NO production and reduced IL-1, IL-6, TNF- $\alpha$ and PGE2 expression. Int J Food Sci Technol 2020;55:1255-64.

[20] Kim KM, Kim YS, Lim JY, Min SJ, Ko HC, Kim SJ, et al. Intestinal anti-inflammatory activity of Sasa quelpaertensis leaf extract by suppressing lipopolysaccharide-stimulated inflammatory mediators in intestinal epithelial Caco-2 cells co-cultured with RAW 264.7 macrophage cells. Nutr Res Pract 2015;9:3-10.

[21] Li H, Feng C, Fan C, Yang Y, Yang X, Lu H, et al. Intervention of oncostatin $\mathrm{M}$-driven mucosal inflammation by berberine exerts therapeutic property in chronic ulcerative colitis. Cell Death Dis 2020;11:271.

[22] Guzman JR, Koo JS, Goldsmith JR, Mühlbauer M, Narula A, Jobin C. Oxymatrine prevents NF- $\mathrm{kB}$ nuclear translocation and ameliorates acute intestinal inflammation. Sci Rep 2013;3:1629.

[23] Liu N, Zhang GX, Niu YT, Wang Q, Zheng J, Yang JM, et al. Anti-inflammatory and analgesic activities of indigo through regulating the $\mathrm{IKK} \beta / \mathrm{I} \kappa \mathrm{B} / \mathrm{NF}-\kappa \mathrm{B}$ pathway in mice. Food Funct 2020;11:8537-46.

[24] Pandurangan AK, Mohebali N, Hasanpourghadi M, Looi CY, Mustafa MR, Mohd Esa N. Boldine suppresses dextran sulfate sodium-induced mouse experimental colitis: NF- $\kappa \mathrm{B}$ and IL-6/ STAT3 as potential targets. Biofactors 2016;42:247-58.

[25] Zhou Z, Bian C, Luo Z, Guille C, Ogunrinde E, Wu J, et al. Progesterone decreases gut permeability through upregulating occludin expression in primary human gut tissues and Caco-2 cells. Sci Rep 2019;9:8367.

[26] Tani N, Sugita K, Yanagihara S, Yamamoto O. Infliximab-induced bullous pemphigoid and anti-desmoglein 3 and anti-BP180 autoantibodies in a patient with ulcerative colitis. Eur J Dermatol 2019;29:88-90.

[27] Benites BD, Gilli SCO, Saad STO. Obesity and inflammation and the effect on the hematopoietic system. Rev Bras Hematol Hemoter 2014;36:147-51.

[28] Lee SH, Lee HR, Kwon JY, Jung K, Kim SY, Cho KH, et al. A20 ameliorates inflammatory bowel disease in mice via inhibiting

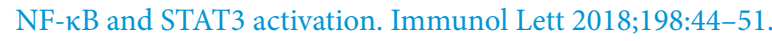

